\title{
Treatment Outcomes of Early Carcinoma Cervix Before and After Sub Specialisation
}

\author{
Vinotha Thomas*, Dhanya Thomas,* Ajit Sebastian*, Anitha Thomas*,
}

Rachel Chandy*, Thomas Samuel Ram\#, Ramani Manoj Kumar \$, Abraham Peedicayil*

\section{Introduction}

Christian Medical College, Vellore, Tamil Nadu, India

- The benefit of gynecologic oncology sub specialisation in cervical cancer has not been examined as yet.

- This study aims to compare the treatment outcomes in carcinoma cervix before and after gynecologic oncology sub specialisation in a tertiary care hospital, in India.

\section{Methods}

- A retrospective cohort study comparing women with operable cervical cancer who underwent radical hysterectomy before and after gynecologic oncology sub specialisation

- Electronic medical records of women operated for early carcinoma cervix between 2001- 2010 (59) and 2011- 2015 (74) were reviewed. FIGO 2009 staging system was used.

\section{Results}

Table 1: Clinical characteristics

\begin{tabular}{|l|c|c|}
\hline \multicolumn{1}{|c|}{ Clinical characteristics } & 2001-2010 (59) & 2011-2015 ( 74) \\
\hline Median age & $46(26-81)$ & $48.5(28-76)$ \\
\hline Median number of pregnancies & $3(1-10)$ & $3(0-7)$ \\
\hline Clinical stage (FIGO 2009) & & \\
1 a2 & $6(10.2 \%)$ & $5(6.7 \%)$ \\
1b1 & $45(76.3 \%)$ & $63(84 \%)$ \\
1b2 & $4(6.8 \%)$ & $4(5.3 \%)$ \\
IIa1 & $4(6.8 \%)$ & $2(2.7 \%)$ \\
\hline Pre op imaging & $8(14 \%)$ & $41(55 \%)$ \\
MRI* & $14(24 \%)$ & $8(11 \%)$ \\
CT & $25(42 \%)$ & $9(12 \%)$ \\
USG & $12(20 \%)$ & $16(22 \%)$ \\
None & & \\
\hline *statistically significant, p-value $=0.000001$ &
\end{tabular}

Table 3: Follow up

\begin{tabular}{|l|c|c|}
\multicolumn{1}{|c|}{ Characteristic } & 2001-2010 ( 59) & 2011-2015 ( 74) \\
\hline Recurrence & $8(14 \%)$ & $11(15 \%)$ \\
Local & $6(10 \%)$ & $7(10 \%)$ \\
Distant & $1(2 \%)$ & $3(4 \%)$ \\
Local and distant & $1(2 \%)$ & $1(1 \%)$ \\
Progressive disease & $5(9 \%)$ & $2(3 \%)$ \\
No recurrence & $39(66 \%)$ & $55(74 \%)$ \\
No follow up & $7(12 \%)$ & $6(8 \%)$ \\
\hline Overall survival rate at 3 years & $36(61 \%)$ & $48(65 \%)$ \\
Died & $8(14 \%)$ & $6(8 \%)$ \\
Unknown & $15(25 \%)$ & $20(27 \%)$ \\
\hline Recurrence free survival at 3 years & $35(60 \%)$ & $45(61 \%)$ \\
No follow up for 3 years & $13(22 \%)$ & $17(23 \%)$ \\
\hline
\end{tabular}

Table 2: Surgical-pathological characteristics , complications and adjuvant treatment

\begin{tabular}{|c|c|c|}
\hline Clinical characteristics & 2001-2010 (59) & 2011-2015 ( 74) \\
\hline Mean size of tumour $(\mathrm{cm})$ & $3.06(0.5-9)$ & $2.98(0.2-10)$ \\
\hline Histology & & \\
\hline Squamous & $43(73 \%)$ & $57(77 \%)$ \\
\hline Adenocarcinoma & $12(20 \%)$ & $15(20.3 \%)$ \\
\hline Others & $4(7 \%)$ & $2(3 \%)$ \\
\hline $\begin{array}{l}\text { Median number of pelvic nodes } \\
\text { removed }\end{array}$ & $10(2-26)$ & $13(4-26)$ \\
\hline $\mathrm{LN}$ involvement & $10(17 \%)$ & $17(23 \%)$ \\
\hline Vaginal margin nvolved & $1(1.7 \%)$ & $3(4.1 \%)$ \\
\hline Parametrial involvement & $5(8.55 \%)$ & $5(6.8 \%)$ \\
\hline LVSI & $\begin{array}{c}\text { Present }(13: 22 \%) \\
\text { Not reported }(39: \mathbf{6 6 \%})\end{array}$ & $24(32.4 \%)$ \\
\hline Deep stromal invasion & $37(63 \%)$ & $44(59.5 \%)$ \\
\hline Intraoperative complications ${ }^{\#}$ & $8(14 \%)$ & $2(3 \%)$ \\
\hline Blood loss $>1500 \mathrm{ml}$ & 3 & 1 \\
\hline Bowel injury & 1 & - \\
\hline Ureteric injury & 2 & - \\
\hline Pelvic vessel injury & 2 & 1 \\
\hline Post op complications $\$$ & $17(46 \%)$ & $11(15 \%)$ \\
\hline Urological & 13 & 4 \\
\hline Infections & 12 & 7 \\
\hline Pulmonary & 1 & \\
\hline Thromboembolism & 1 & \\
\hline Adjuvant treatment* & $14 / 59(24 \%)$ & $37 / 74(50 \%)$ \\
\hline Radiation & $14(24 \%)$ & $18(24 \%)$ \\
\hline Chemoradiation & - & $16(22 \%)$ \\
\hline Radiation +chemo & - & $3(4 \%)$ \\
\hline Defaulted & $10(17 \%)$ & $6(8 \%)$ \\
\hline None & $35(60 \%)$ & $31(42 \%)$ \\
\hline $\begin{array}{l}\text { \# statistically significant: p-value } \\
\text { \$ statistically significant :p-value } \\
\text { *statistically significant: } p \text {-value }\end{array}$ & $\begin{array}{l}=0.018 \\
=0.0004 \\
=0.000967 .\end{array}$ & \\
\hline
\end{tabular}

\section{Conclusion}

Sub specialisation in gynecologic oncology resulted in increased utilization of radiological services, decreased intraoperative and postoperative complications and improved pathological reporting with appropriate tailoring of adjuvant therapy.

However, increased rates of adjuvant treatment did not translate into better survival. 